\title{
Cloud Computing Models and their Application in LTE based Cellular Systems
}

\author{
A. J. Staring \\ University of Twente \\ P.O. Box 217, 7500AE Enschede \\ The Netherlands \\ a.j.staring@student.utwente.nl
}

\author{
G. Karagiannis \\ University of Twente \\ P.O. Box 217, 7500AE Enschede \\ The Netherlands \\ g.karagiannis@utwente.nl
}

\begin{abstract}
As cloud computing emerges as the next novel concept in computer science, it becomes clear that the model applied in large data storage systems used to resolve issues coming forth from an increasing demand, could also be used to resolve the very high bandwidth requirements on access network, core network equipment and long communication paths between users and servers in the today's cellular networks. The cloud computing model can be used to avoid bottlenecks, better utilize available resources, and minimize delay. This paper analyses how three deployed major cloud computing platforms, i.e., OpenStack, Eucalyptus and OpenNebula could support this. The analysis showed that none of the studied currently deployed cloud computing platforms can satisfy the derived requirements without enhancing and extending the functions and modules supported by each platform.
\end{abstract}

Index Terms-Cloud computing platforms, Network virtualisation / cloudification, cellular systems, LTE

\section{INTRODUCTION}

Cloud computing is one of the most discussed and promising fields in modern computer science. Contrary to common belief cloud computing is not a form of implementation, but a model. Applying the cloud computing model to a system or part of a system can be denoted as cloudification. The implementation of the cloud computing model is typically denoted as virtualisation. The model used in this paper is defined by the National Institute for Standards and Technology (NIST) and states:

Cloud computing is a model for enabling ubiquitous, convenient, on-demand network access to a shared pool of configurable computing resources (e.g., networks, servers, storage, applications, and services) that can be rapidly provisioned and released with minimal management effort or service provider interaction [1].

The cloud computing model is mostly implemented in large data storage systems, providing Infrastructure-as-a-Service (IaaS), Platform-as-a-Service (PaaS) and/or Software-as-aService (SaaS) service models.

IaaS provides customers processing, storage, network and other fundamental computing resources on which they can run software. This includes operating systems and applications. This is done by providing the customer with a virtual machine. A virtual machine (VM) is a software implementation of a machine/computer and runs in much the same way as a physical machine. The idea is that multiple VMs can run on a single physical machine and that the software running inside the virtual machine is limited to the resources and abstractions provided by the virtual machine and cannot break out of its virtualised environment.

PaaS is using the services provided by IaaS, and offers customers an environment for application development, testing, deployment and hosting without the complexity of managing the underlying infrastructure and supporting software. To facilitate the customer in such an environment the provider can support various programming languages, libraries, services and tools.

$\mathrm{SaaS}$ is using the services provided by PaaS and delivers its customers on-demand applications running on a cloud computing platform. The applications are accessible, mostly over the Internet, through various clients like web browsers or thin clients. It is also possible to access the application through a programmable interface (API). SaaS customers do not have to be concerned about the underlying infrastructure and have no influence on the application features, apart from the user specific configuration settings.

When providing IaaS, virtual machines are connected to each other in a virtual network. This is done by virtualising network components like cables, switches, routers and firewalls. This virtual network can be made as simple or complex as one can imagine [2], [3]. Network virtualisation in large date storage systems virtualises wired networks. There is no practical need to virtualise a wireless network in such systems.

In the world of today, the increasing use of smartphones, tablets and other portable devices using the cellular system, requires from the mobile cellular system to cope with this increasing demand. One of the most advanced cellular systems (i.e., 4th generation) being currently under specification and standardisation by 3GPP (Third Generation Partnership Project) is the Long Term Evolution (LTE) system, see [4], [5]. An LTE network consists of two main parts, the Evolved UMTS Terrestrial Radio Access Network (E-UTRAN) and the Evolved Packet Core (EPC) network. The typical E-UTRAN consists only of evolved NodeBs (eNodeB), which represent the Base Stations (BS) used to provide radio access to all Mobile Stations that are within their radio coverage. The EPC 
grants users: (1) permission to the LTE based cellular system, (2) support for multimedia service connectivity, roaming and mobility. EPC consists of several network entities such as: (a) the Mobility Management Entity (MME) that supports the mobility management, (b) the Home Subscriber Server (HSS) that maintains the subscription profiles of each user, (c) the Packet Data Network Gateway (PGW) that represents the packet data network gateway to the Internet, (d) the Serving Gateway (SGW) that manages the user data tunnels between the eNodeBs and the PGW, under the supervision of the MME.

Today's mobile cellular networks are highly centralised and not optimised for high-volume data applications, which will evolve with 4G (e.g., LTE) and beyond technologies. Operators typically use centralised network architectures that lead to very high bandwidth requirements on core network equipment and long communication paths between users and servers, which wastes network resources and increases delay. Shared distributed mobile network architectures, are needed to avoid bottlenecks, better utilise available resources, and minimise delay.

The cloud computing model could be applied in mobile cellular systems to solve this issue by offering decentralised computing, smart storage, on-demand, elastic and Pay-asyou-Go services to third party operators and users, see e.g. [6]. There are a few papers available describing how virtual telecommunication operators are using the cloud computing model to provide their services, see [7], [8]. There are also papers that focus on how wireless networking can be virtualised [9], [10], and even ones on the virtualisation of a cellular system, see e.g., [11], [12].

However, not many research activities focused on how existing cloud computing platforms can be used to cloudify LTE based cellular systems (including the E-UTRAN, EPC, and Operators services, e.g., IMS (IP Multimedia Subsystems)). This paper aims to resolve this dilemma. So the main research question is:

\section{"How can the cloud computing model be applied in LTE cellular systems?"}

In order to answer the main research question the research is broken down in sub questions.

1) What are the main differences between applying network cloudification in (1) large data storage systems and (2) LTE based cellular systems?

2) Which challenges have to be solved when using current cloud computing platforms in LTE based cellular systems?

Research question (1) will be answered using literature study. The literature study will provide a list of challenges that current cloud computing platforms [13], [14], [15] need to resolve in order to apply the cloud computing model in LTE based cellular systems. Research question (2) will be answered by defining the main requirements needed to implement the cloud computing model in LTE based cellular systems and by studying how these requirements are satisfied by the specifications of the currently deployed cloud computing platforms. In order to analyse cloud computing platforms for their ability to be used in cloudifying LTE based cellular systems, criteria (requirements) need to be established. The criteria (requirements) are derived within the context of the EU FP7 Mobile Cloud Networking project [6], [16]. These criteria can be used to measure the cloud computing platforms ability to cloudify the LTE based cellular system (including E-UTRAN, EPC, and Operators services, e.g., IMS). By researching the cloud computing platforms documentation, it will become clear whether these criteria can be met or not. These criteria are:

1) Support virtualised foundational infrastructural resources from the Radio Access Network and Mobile Core Network and Data Centre

2) Support a coordination and orchestration function from the infrastructure perspective for the composition, provisioning, life cycle management of services and maintenance of agreed SLA (Service Level Agreements) guarantees spread across Radio Access Network and Core Network.

3) Support monitoring facilities for extraction, preprocessing, distribution, storage, analysis and notification of metrics such that higher-level services can utilise these facilities to enable adaptive platform services (such as SLAs), where certain guarantees and service quality (QoS) and/or experience (QoE) must be maintained

4) Support mechanisms to deliver virtual resources as service for the execution of non-traditional virtualised instances such as virtualised eNodeB, EPC (e.g., MME, PGW, SGW) and IMS functionality

5) Support unified and consistent interfaces on top of and between disparate cloud computing physical infrastructure management frameworks (i.e., Radio Access Network, Mobile Core Network, Data centre)

The analysis will be done using the documentation of the latest available version of the cloud computing platforms, see Table I.

TABLE I

Cloud COMPUTING PLATFORM VERSION AND RELEASE DATE

\begin{tabular}{|l|l|l|}
\hline Platform & Version & Release date \\
\hline \hline OpenStack [13] & 2012.1 (Essex) & $04-05-2012$ \\
\hline Eucalyptus [14] & 2.0 .3 & $25-05-2011$ \\
\hline OpenNebula [15] & 3.4 .1 (Wild Duck) & $03-05-2012$ \\
\hline \hline
\end{tabular}

This paper is organized as follows. Section 2 discusses three major cloud computing platforms, i.e., OpenStack, Eucalyptus and OpenNebula. Section 3 analyses how the derived criteria (requirements) are satisfied by the specifications of these three major cloud computing platforms. Furthermore, section 3 answers research questions (1) and (2). Finally, section 4 concludes and provides recommendations for future work. 


\section{CLOUd COMPUTING PLATFORMS}

This section discusses three major cloud computing platforms, which are OpenStack, Eucalyptus and OpenNebula.

1) OpenStack: OpenStack Essex, [13] the fifth version of OpenStack, focuses on quality, usability and extensibility across enterprise, service provider and high performance computing deployments. OpenStack Essex allows users to leverage pools of

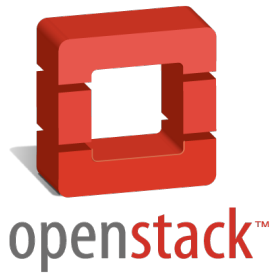
on-demand, self-managed compute, storage and networking resources to build efficient, automated private and public cloud infrastructures.

OpenStack Essex includes, but is not limited to, the following modules:

Compute: The OpenStack cloud operating system enables service providers to offer on-demand computing resources, by provisioning and managing large networks of virtual machines. Compute resources are accessible via APIs for developers building cloud applications and via web interfaces for administrators and users. The Compute architecture is designed to scale horizontally. OpenStack is designed to provide flexibility and to manage and automate pools of Compute resources and can work with multiple supported hypervisors in a virtualised environment like KVM (Kernel-based Virtual Machine) and Xen. Linux container technology such as LXC (Linux Containers) is also supported for scenarios where users need to minimise virtualisation overhead and gain greater efficiency and performance. In addition to different hypervisors, OpenStack supports ARM (Advanced RISC-Reduced Instruction Set Computer Machine) and alternative hardware architectures.

Object Storage: OpenStack provides redundant, scalable object storage using clusters of standardized servers capable of storing petabytes of data. Object Storage is not a traditional file system, but rather a distributed storage system for static data such as virtual machine images, photo storage, email storage, backups and archives. Having no central "brain" or master point of control provides greater scalability, redundancy and durability. Objects and files are written to multiple disk drives spread across servers in the data centre, with the OpenStack software responsible for ensuring data replication and integrity across the cluster. Storage clusters scale horizontally simply by adding new servers.

Block Storage: OpenStack also provides persistent block level storage devices for use with OpenStack compute instances. The Block Storage system manages the creation, attaching and detaching of the block devices to servers. Block Storage volumes are fully integrated into OpenStack Compute and the Dashboard allowing for cloud users to manage their own storage needs. In addition to using simple Linux server storage, it has unified storage support for numerous storage platforms. Block Storage is appropriate for performance sensitive scenarios such as database storage, expandable file systems, or providing a server with access to raw block level storage.
Networking: OpenStack Networking is a pluggable, scalable and API-driven system for managing networks and IP addresses. OpenStack provides flexible networking models to suit the needs of different applications or user groups. Standard models include flat networks or VLANs for separation of servers and traffic. OpenStack Networking manages IP addresses, allowing for dedicated static IPs or DHCP (Dynamic Host Configuration Protocol). Floating IPs allow traffic to be dynamically rerouted to any of the Compute resources, which allows to redirect traffic during maintenance or in the case of failure. Users can create their own networks, control traffic and connect servers and devices to one or more networks. Administrators can take advantage of softwaredefined networking (SDN) technology like OpenFlow to allow for high levels of multi-tenancy and massive scale. OpenStack Networking has an extension framework allowing additional network services, such as intrusion detection systems (IDS), load balancing, firewalls and virtual private networks (VPN) to be deployed and managed.

Dashboard: The OpenStack Dashboard provides administrators and users a graphical interface to access, provision and automate cloud-based resources. The extensible design makes it easy to plug in and expose third party products and services, such as billing, monitoring and additional management tools. Developers can automate access or build tools to manage their resources using the OpenStack API or the Amazon EC2 (Elastic Compute Cloud) compatibility API. The dashboard is an extensible web app that allows cloud administrators and users to control their compute, storage and networking resources. As a cloud administrator, the dashboard provides an overall view of the size and state of the cloud and is able to create users and projects, assign users to projects and set limits on the resources for those projects. The Dashbord provides users a self-service portal to provision their own resources within the limits set by administrators.

2) Eucalyptus: Eucalyptus [14] is a EUCALYPTUS widely deployed software platform for private IaaS clouds. It uses existing infrastructures to create a scalable, secure web services layer that abstracts compute, network and storage to offer IaaS. Eucalyptus takes advantage of modern infrastructure virtualisation software to create elastic pools that can be dynamically scaled up or down depending on application workloads. Eucalyptus web services are uniquely designed for hybrid clouds using the industry standard Amazon Web Services (AWS) API.

Eucalyptus key functionality includes:

Self-service User Portal: The Eucalyptus User Console provides an interface for users to self-service provision and configure compute, network, and storage resources. Development and test teams can flexibly and securely manage their virtual instances using built-in key management and encryption capabilities. Access to virtual instances is available using familiar SSH (Secure Shell) and RDP (Remote Desktop Protocol) mechanisms. Virtual instances with application configuration can be stopped and re-started securely using encrypted boot from Elastic Block Storage (EBS) capability. 
High Availability Cloud IaaS: Systems failure is expected and unavoidable in a data center, but Eucalyptus has been designed to be highly available at multiple levels. IaaS service components Cloud Controller (CLC), Cluster Controller (CC), Walrus, Storage Controller (SC) and VMware Broker (VB) are configurable as redundant systems that are very resilient to multiple types of failures. Management state of the cloud machine will be preserved and reverted to normal operating conditions in the event of a hardware or software failure.

Heterogeneous Hypervisor Management: Build and manage multiple hypervisor cluster environments in an IaaS cloud. Manage existing VMware vSphere, VMware ESX (Elastic Sky $\mathrm{X})$, VMware ESXi, KVM, and Xen virtual environments from a single pane as Amazon AWS compatible Eucalyptus hybrid clouds.

Manage Multiple Machine Image Formats: Eucalyptus can run multiple versions of Microsoft Windows and Linux virtual machine images on IaaS clouds and build a library of Eucalyptus Machine Images (EMIs) with application metadata that are decoupled from infrastructure details to allow them to run on Eucalyptus clouds. Amazon Machine Images are also compatible with Eucalyptus clouds. VMware Images and VMware vApps can be converted to run on Eucalyptus clouds, and Amazon AWS and Amazon compatible public clouds.

Scriptable Architecture: Production environments want flexible, configurable and scriptable system to automate as many functions as possible in a cloud management platform. Users have the choice of integrating web services API with their preferred vendor management systems or using an Amazon AWS compatible management stack to manage Eucalyptus clouds. All the functionality is exposed in a scriptable interface to work with other system management tools.

Identity Management: Eucalyptus user identity management can be integrated with existing Microsoft Active Directory or LDAP (Lightweight Directory Access Protocol) systems to have fine-grained role based access control over cloud resources. Flexible Accounting, Chargeback, and Quota Management Define and allocate resource quotas for the users and groups with Eucalyptus 3's quota management features. Control resource allocation across clusters, defined by users and groups.

Accounting, Chargeback, and Quota Management: Define and allocate resource quotas for users and groups with quota management features. Control resource allocation across clusters, defined by users and groups and analyse cloud usage patterns by using resource accounting module. Compute and storage usage data are available in various formats for visualization, reporting, and analysis by business systems for both enterprises and integration with chargeback and billing platforms.

Resource Administration And Console: The Eucalyptus Dashboard provides cloud administrators with a graphical console for performing several cloud management tasks including all virtual and physical resource management and virtual cloud resource configuration, provisioning and de-provisioning.
OpenNebula.org

3) OpenNebula: OpenNebula [15] is a cloud computing toolkit for managing heterogeneous distributed data centre infrastructures. The OpenNebula toolkit manages a data centre virtual infrastructure to build private, public and hybrid IaaS clouds. OpenNebula orchestrates storage, network, virtualisation, monitoring, and security technologies to deploy multi-tier services (e.g. compute clusters) as virtual machines on distributed infrastructures, combining both data centre resources and remote cloud resources, according to allocation policies.

OpenNebula key features include:

User Security Management: Secure and efficient users and groups pluggable subsystem for authentication and authorisation of requests with complete functionality for user management. The authorisation framework allows multiplerole support for different types of users and administrators, delegated control to authorised users, secure isolated multitenant environments, and easy resource (VM template, VM image, VM instance, virtual network and host) sharing. Administrators have complete functionality for management of grouped users where each group has configurable access to shared resources so enabling a multi-tenant environment with multiple groups sharing the same infrastructure.

On-demand Provision of Virtual Data Centres: A Virtual Data Centres (VDC) is a fully-isolated virtual infrastructure environment where a group of users, under the control of the VDC administrator, can create and manage compute, storage and networking capacity. There is also support for the creation and management of multiples VDCs within the same logical cluster and zone.

Control and Monitoring of Virtual Infrastructure: OpenNebula has a Storage and Repository Subsystem with functionality for VM image and template management. The platform also has full control of VM instance life-cycle and functionality for VM instance management. OpenNebula also has broad network virtualisation capabilities with traffic isolation, ranged or fixed networks, definition of generic attributes to define multi-tier services consisting of groups of inter-connected VMs, and functionality for virtual network management to interconnect VM instances. The tagging of users, VM images and virtual networks with arbitrary metadata that can be later used by other components and API's future use.

Virtual Machine Configuration: Complete definition of VM attributes and requirements and the support for automatic configuration of VMs with advanced contextualization mechanisms with a wide range of guest operating system including Microsoft Windows and Linux. For networking, OpenNebula offers flexible network definition including configuration of firewalls for VMs to specify a set of black/white TCP / UDP ports.

Control and Monitoring of Physical Infrastructure: OpenNebula is able to deploy public, private and hybrid clouds. The Host Management Subsystem offers functionality for management of physical hosts with the dynamic creation of clusters as a logical set of physical resources, namely: hosts, networks and data stores, within each zone. OpenNebula has a powerful and extensible built-in monitoring subsystem and re- 
source quota management to allocate, track and limit resource utilisation. The virtualisation Subsystem supports a wide range of hypervisors like Xen, KVM and VMware ESX, centralised management of environments with multiple hypervisors, and support for multiple hypervisors within the same physical box. Storage Subsystem with support for multiple data stores to balance I/O operations between storage servers, or to define different SLA policies (e.g. backup) and performance features for different VM types or users.

Standard Cloud Interfaces and Self-Service Portal: OpenNebula is able to simultaneously expose multiple cloud APIs including the AWS EC2 API service for compatibility with EC2 ecosystem tools and client tools. OpenNebula also has a self-service provisioning portal to allow non-IT end users to easily create, deploy and manage compute, storage and network resources and a Unix-like Command Line Interface to manage all resources: users, VM images, VM templates, VM instances, virtual networks, zones, VDCs, physical hosts, accounting, authentication, authorisation for cloud administrators. The modular and extensible Graphical Interface providing usage graphics and statistics with cloudwatch-like functionality, VNC support, different system views for different roles, catalogue access and multiple-zone management.

\section{Analysis of Applying Existing Cloud COMPUTING PlatForms IN LTE BASED CELLULAR SYSTEMS AND CHALLENGES}

The analysis starts by mapping the features and functionality of the cloud computing platforms to the criteria introduced in Section 1. The analysis can be found in Table II, III and IV showing the criteria and the modules which could be used to support each criterion. The criteria are numbered according to the numbering used in Section 1.

It is important to be noticed that the functions/modules (including the APIs) supported by the three cloud computing platforms are able to support wired and fixed virtualisation scenarios that are making use of large data centres as well as that all three cloud computing platforms have support for billing and charging using a Pay-as-you-go policy which can be integrated by their API into other financial systems of the operator.

These functions/modules (including the APIs) are not designed and implemented to support virtualised scenarios where the allocation and release of E-UTRAN, EPC and Operators services (e.g., IMS) resources takes place in a very dynamic and fast changing environment.

Therefore, in order to support the five criteria described in Section 1, these functions/modules (including the APIs) need to be enhanced such that they can support challenges, such as dynamic network access connectivity and release, QoS support, cross-operator dynamic mobility and roaming management, security and privacy support, billing and charging using a Pay-as-you-go policy for an environment where the allocation and release of E-UTRAN, EPC and Operators services (e.g., IMS) resources takes place in a very dynamic and fast changing environment.
TABLE II

ANALYSIS OPENSTACK

\begin{tabular}{|c|l|}
\hline Criterion & Modules to be enhanced \\
\hline \hline 1 & Compute and Networking module \\
\hline 2 & Compute and Networking module \\
\hline 3 & Dashboard \\
\hline 4 & Compute and Networking module \\
\hline 5 & Dashboard \\
\hline
\end{tabular}

TABLE III

ANALYSIS EUCALYPTUS

\begin{tabular}{|c|l|}
\hline Criterion & Modules to be enhanced \\
\hline \hline 1 & $\begin{array}{l}\text { Heterogeneous Hypervisor Management, Manage } \\
\text { Multiple Machine Image Formats and High Avail- } \\
\text { ability Cloud IaaS }\end{array}$ \\
\hline 2 & $\begin{array}{l}\text { Self-service User Portal, Resource Administration } \\
\text { And Console and Identity Management }\end{array}$ \\
\hline 3 & $\begin{array}{l}\text { Self-service User Portal, Resource Administration } \\
\text { And Console and Identity Management }\end{array}$ \\
\hline 5 & $\begin{array}{l}\text { Scriptable Architecture, Heterogeneous Hypervisor } \\
\text { Management, Manage Multiple Machine Image For- } \\
\text { mats and High Availability Cloud IaaS }\end{array}$ \\
\hline 5 & $\begin{array}{l}\text { Scriptable Architecture, Self-service User Portal, } \\
\text { Resource Administration And Console and Identity } \\
\text { Management }\end{array}$ \\
\hline \hline
\end{tabular}

It needs to be noted that depending on the operators requirements, the use of additional not in this paper discussed functions/modules could be used. However, those additional modules probably also need to be enhanced in order to support the criteria given in Section 1.

Table II, III and IV show the criteria and the modules of OpenStack, Eucalyptus and OpenNebula, respectively, which need to be enhanced in order to support these criteria. Furthermore, these tables show that enhancements and extensions need to be made in order to support challenges, such as dynamic network access connectivity and release, QoS support, cross-operator dynamic mobility and roaming management, security and privacy support, billing and charging using a Pay-as-you-go policy for an environment where the allocation and release of E-UTRAN, EPC and Operators services (e.g., IMS) resources take place in a very dynamic and fast changing environment for all three cloud computing platforms.

\section{CONClusions AND Future Work}

This paper investigated how the cloud computing model could be applied into the LTE (Long Term Evolution) cellular system and analyses how three deployed major cloud computing platforms could support this.

Section 2 discusses three major current cloud computing platforms, i.e., OpenStack, Eucalyptus and OpenNebula. Section 3 answers research questions (1) and (2) by analysing how the main requirements needed to implement the cloud computing model in LTE based cellular systems (including the E-UTRAN, EPC, and Operators services, e.g., IMS) are satisfied by the specifications of the currently deployed cloud computing platforms. 
TABLE IV

ANALYSIS OPENNEBULA

\begin{tabular}{|c|l|}
\hline Criterion & Modules to be enhanced \\
\hline \hline 1 & $\begin{array}{l}\text { Control and Monitoring of Physical Infrastructure } \\
\text { and On-demand Provision of Virtual Data Centres }\end{array}$ \\
\hline 2 & $\begin{array}{l}\text { Control and Monitoring of Virtual Infrastructure and } \\
\text { Virtual Machine Configuration }\end{array}$ \\
\hline 3 & $\begin{array}{l}\text { User Security Management and Standard Cloud In- } \\
\text { terfaces and Self-Service Portal }\end{array}$ \\
\hline 5 & $\begin{array}{l}\text { On-demand Provision of Virtual Data Centres, Con- } \\
\text { trol and Monitoring of Virtual Infrastructure and } \\
\text { Virtual Machine Configuration }\end{array}$ \\
\hline $\begin{array}{l}\text { User Security Management } \text { and Standard Cloud In- } \\
\text { terfaces and Self-Service Portal }\end{array}$ \\
\hline
\end{tabular}

In particular, this paper showed that none of the three major current cloud computing platforms fully satisfy the criteria from Section 1, as the most of the existing functions/modules cannot support the challenges that need to be fulfilled by scenarios where the allocation and release of E-UTRAN, EPC and Operators services (e.g., IMS) resources takes place in a very dynamic and fast changing environment. It is expected that these criteria could be satisfied if, at least, the functions/modules listed in Table II, III, IV are enhanced in order to fulfil challenges, such as dynamic network access connectivity and release, QoS support, cross-operator dynamic mobility and roaming management, security and privacy support, billing and charging using a Pay-as-you-go policy for an environment where the allocation and release of E-UTRAN, EPC and Operators services (e.g., IMS) resources takes place in a very dynamic and fast changing environment.

The functions/modules of the cloud computing platforms OpenStack, Eucalyptus and OpenNebula could satisfy several of the derived criteria after solving several challenges. Once enhancements have been made in the above specified functions/modules, the enhanced cloud computing platforms could be used to enable the realisation of the cloudification of the LTE based cellular system.

This paper analysed how the three cloud computing platforms can be applied in an LTE based cellular system, without providing a comparison between the three cloud computing platforms. Such a comparison is considered to be a future work item.

\section{ACKNOWLEDGMENTS}

This work is accomplished in the context of the Mobile Cloud Networking (MCN) project. We therefore, would like to acknowledge the European Commission, since the MCN project is an EC co-funded Integrated Project under the $7^{\text {th }}$ RTD Framework Programme, FP7-ICT-2011-8 - number 318109 .

\section{REFERENCES}

[1] P. Mell and T. Grance, "The NIST Definition of Cloud Computing," National Institute of Standards and Technology (NIST), Special Publication 800-14, September 2011.

[2] N. M. Mosharaf Kabir Chowdhury and R. Boutab, "Network Virtualization: State of the Art and Research Challenges," IEEE Communications Magazine, pp. 20-26, July 2009.
[3] N. Chowdhury and R. Boutaba, "A survey of network virtualization," Computer Networks, vol. 54, no. 5, pp. 862-876, 2010.

[4] 3GPP, "LTE specifications," http://www.3gpp.org/lte, visited in June 2012.

[5] 3GPP TS 23.401, "General Packet Radio Service (GPRS) enhancements for Evolved Universal Terrestrial Radio Access Network (E-UTRAN) access (Release 11)," 3GPP, Technical Specification TS 23.401, V11.1.0, March 2012.

[6] European FP7 Integrated Project, "Mobile Cloud Networking," FP7-ICT2011-8, No. 318109, started in November 2012.

[7] P. Bosch, A. Duminuco, F. Pianese, and T. L. Wood, "Telco clouds and Virtual Telco: Consolidation, convergence, and beyond," in Proceedings of the 12th IFIP/IEEE International Symposium on Integrated Network Management, IM, 2011, pp. 982-988.

[8] S. Hall, "Evolution of telco services utilising Infrastructure-as-a-Service (IaaS)," in 2011 15th International conference on Intelligence in Next Generation Networks, ICIN 2011, 2011, pp. 247-252.

[9] S. Zhou, M. Zhao, X. Xu, J. Wang, and Y. Yao, "Distributed wireless communication system: A new architecture for future public wireless access," IEEE Communications Magazine, vol. 41, no. 3, pp. 108-113, 2003.

[10] Z. Zhu, P. Gupta, Q. Wang, S. Kalyanaraman, Y. Lin, and H. Franke, "Virtual base station pool: Towards a wireless network cloud for radio access networks," in Proceedings of the 8th ACM International conference on Computing Frontiers, CF'11, 2011.

[11] Y. Zaki, M. A. Khan, L. Zhao, and C. Görg, "Realizing the Broker Based Dynamic Spectrum Allocation through LTE Virtualization and Uniform Auctioning," Proc. of IFIP NETWORKING 2011 Workshop, vol. 6827 LNCS, pp. 85-97, 2011.

[12] L. Zhao, M. Li, Y. Zaki, A. Timm-Giel, and C. Görg, "LTE virtualization: From theoretical gain to practical solution," in Proceedings of the 23rd International Teletraffic Congress, ITC 2011, 2011, pp. 71-78.

[13] Rackspace Cloud Computing, "OpenStack Open Source Cloud Computing Software," http://www.openstack.org, visited in June 2012.

[14] Eucalyptus, "Cloud Computing Software," http://www.eucalyptus.com, visited in June 2012.

[15] OpenNebula, "The Open Source Solution for Data Center Virtualization," http://www.opennebula.org, visited in June 2012.

[16] Mobile Cloud Networking (MCN), "Official website of the EU FP7 Mobile Cloud Networking project," https://www.mobile-cloud-networking. eu, visited in March 2013. 